\title{
The Effect of Antenna Polarization and Body Morphology on the Measurement Uncertainty of a Wearable Multi-Band Distributed Exposure Meter
}

\author{
Reza Aminzadeh • Arno Thielens • \\ Sam Agneessens • Patrick Van Torre • \\ Matthias Van den Bossche • Stefan Dongus • \\ Marloes Eeftens • Anke Huss • Roel Vermeulen · \\ René de Seze - Paul Mazet • Elisabeth Cardis • \\ Hendrik Rogier · Martin Röösli · Luc Martens · \\ Wout Joseph
}

Received: date / Accepted: date

\begin{abstract}
This paper studies the effect of antenna polarization on measurement uncertainty of a multi-band body-worn distributed exposure meter (BWDM). The BWDM is a device for assessing electromagnetic fields in real environments accurately. The BWDM consists of 8 nodes and is calibrated on body for simultaneous measurement of the incident power density
\end{abstract}

R. Aminzadeh · A. Thielens $\cdot$ S. Agneessens $\cdot$ P. Van Torre $\cdot$ M. Van den Bossche $\cdot$ H. Rogier $\cdot$ L. Martens W. Joseph

Department of Information Technology, Ghent University/imec, B-9052 Ghent, Belgium

E-mail: [reza.aminzadeh, arno.thielens, sam.agneessens, patrick.vantorre, matthias.vandenbossche, hendrik.rogier, luc1.martens, wout.joseph]@ugent.be

S. Dongus · M. Eeftens · M. Röösli

Department of Epidemiology and Public Health, Swiss Tropical and Public Health Institute, Socinstrasse 57,4051 Basel, Switzerland

University of Basel, Petersplastz 1, 4001 Basel, Switzerland

E-mail: [stefan.dongus, marloes.eeftens, martin.roosli]@swisstph.ch

A. Huss $\cdot$ R. Vermeulen

Institute for Risk Assessment Sciences (IRAS), Utrecht University, 3508 TD Utrecht, The Netherlands

E-mail: [a.huss, r.c.h.vermeulen]@uu.nl

R. de Seze

National Institute for Industrial Environment and Risks (INERIS), Parc Technologique Alata BP2, 60550 Verneuil-en-Halatte, France

E-mail: rene.de-seze@ineris.fr

P. Mazet

Technical Center for Mechanical Industries (CETIM), 60300 Senlis, France

E-mail: paul.mazet@cetim.fr

E. Cardis

Barcelona Institute for Global Health (ISGlobal), 08003 Barcelona, Spain

E-mail: elisabeth.cardis@isglobal.org 
in four frequency bands. Each node contains an antenna that can have two potential antennapolarizations. The BWDM is calibrated on four human subjects in an anechoic chamber to determine its measurement uncertainty in terms of $68 \%$ confidence interval $\left(\mathrm{CI}_{68}\right)$ of the on-body antenna aperture. The results show that using a fixed polarization of the antennas on body can lead to a different $C I_{68}$ up to maximum $4.9 \mathrm{~dB}$ when is worn by another person which is still $9.6 \mathrm{~dB}$ lower than the measurement uncertainty of commercial exposure meters.

Keywords RF exposure - Personal exposure meters · polarization dependency, Body Morphology $\cdot$ Measurement uncertainty

\section{Introduction}

Characterization of exposure to emerging wireless technologies has been identified as an important issue by the World Health Organization (WHO) to be addressed [1]. Personal exposure to radio-frequency (RF) electromagnetic fields (EMFs) is usually measured by personal exposure meters (PEMs) [2-6]. These are portable devices that are worn on body to continuously measure the incident electric fields at the location of a subject wearing the device. These devices are calibrated in free space but are used on body. Therefore, it is questionable whether they can measure the incident EMFs on body accurately. Several studies have shown that PEM's measurements are influenced by the presence of a human body. A review of different sources contributing to uncertainty of PEMs can be found in [7]. Large variations in response of PEMs have been reported up to $35 \mathrm{~dB}$ for single measurement points [8]. The presence of human body shields part of the EMFs (known as body shadowing). This results in an unknown estimation of the actual EMFs $[4,6,9]$. Moreover, PEMs exhibit an unwanted dependence on the polarization of the incident fields [4, 10].

In order to address the above issues, on-body calibration of PEMs has been suggested in previous studies as well as using multiple PEMs and a correction factor to compensate for shielding of the body $[4,6,11,12]$. In addition, personal distributed exposure meters (PDEs) have been proposed for $941 \mathrm{MHz}$ [13] and $2450 \mathrm{MHz}$ [14]. Recently a multi-band body-worn distributed exposure meter (BWDM) measuring at 11 telecommunication bands has been proposed [15]. This distributed exposure meter has been calibrated on body and may have a significantly smaller measurement uncertainty compared to the commercial PEMs [13-15]. To design the PDE and the BWDM, the locations and polarizations of the antennas were determined as those that lead to the lowest measurement uncertainty using the calibrations on human subjects $[15,16]$.

In [15], the effect of antenna location on the measurement uncertainty of a BWDM has been studied. The polarization dependency of a distributed exposure meter and the effect of body morphology on this polarization dependency has not been assessed yet. In addition, the existing literature data are not conclusive regarding polarization dependency and the absorption of the EMFs and thus not a general agreement can be found [17]. Before an exposure meter appears on the market, it is necessary to calibrate it on body since it has been shown that on-body calibration of a PEM compensates the under/over estimation of the actual fields caused by body shielding $[4,6,7,12,14-16]$. Using the on-body calibration, the incident fields in the presence and absence of the body are measured. This results in a correction factor to de-embed the presence of human body from the incident fields perturbed due to the body shadowing.

The goal of this study is to determine how different body morphologies can impact the uncertainty of the measured incident EMFs considering the BWDM is calibrated on a 
limited number of subjects. The novelties of this paper are as follows: 1) For the first time, the polarization dependency of a BWDM and the effect of body morphology on this dependency is assessed. The BWDM consists of 2 nodes for each of the four studied frequency band and contains antennas that can have two potential antenna-polarizations. 2) To determine the measurement uncertainty of a BWDM for both antenna-polarizations for four different subjects (different body morphologies). 3) As an application, an outdoor measurement is performed in a real environment and the results are compared to the measurements of a commercial PEM.

The methodology is described in Section 2. Section 3 presents the results and Section 4 discusses the findings of this study. Finally Section 5 concludes the paper.

\section{Methodology}

This paper follows the design of the BWDM presented in [15]. The considered frequency bands are as follows (see Table 1): The uplink (UL) and downlink (DL) bands of Global System for Mobile Communications (GSM) $900 \mathrm{MHz}$, Wireless Fidelity $2 \mathrm{GHz}$ (WiFi-2G) and DL band of Long-Term Evolution (LTE) $2600 \mathrm{MHz}$.

Table 1 The studied frequency bands. $f_{c}$ is the center frequency.

\begin{tabular}{lll}
\hline Band & Range $(\mathrm{MHz})$ & $f_{c}(\mathrm{MHz})$ \\
\hline 900-UL & $879-915$ & 896 \\
900-DL & $921-960$ & 941 \\
WiFi-2G & $2400-2485$ & 2443 \\
2600-DL & $2620-2690$ & 2655
\end{tabular}

\subsection{Textile antennas}

The design of the used textile antennas has been previously presented in [18]. The design is based on the Substrate Integrated Waveguide technology (SIW) and therefore, benefits from high body-antenna isolation. The substrate and conductive parts are fabricated using closed-cell expanded rubber and copper-coated nylon, respectively. The textile materials are patterned using a $\mathrm{CO}_{2}$ laser with spot size less than $0.1 \mu \mathrm{m}$. Next, the layers are laminated together using thermally activated glue. All the antennas are designed to have an $\left|S_{11}\right|$ (magnitude of the reflection coefficient) smaller than $-10 \mathrm{~dB}$ in each frequency band (see Table 1). Each antenna has a gain of 3 to $5 \mathrm{dBi}$ along the direction away from the body surface. The dimensions of the largest antenna are $110 \times 110 \times 11 \mathrm{~mm}^{3}$. All the antennas are elliptically polarized. The antennas have an axial ratio (AR) in the range of 5 (WiFi-2G) to $15 \mathrm{~dB}(900-\mathrm{DL})$ that is the ratio between the minor and major axis of the polarization ellipse.

\subsection{Receiver nodes}

Figure 1 shows an example of a receiver node integrated into a textile antenna. Each node consists of a textile antenna, which registers the impeding signals. The signal captured by 
the antenna is filtered in the desired band of interest. The filtered signal is then detected by a logarithmic detector chip over a dynamic range of $80 \mathrm{~dB}$. The analogue signals generated by the detector chip are digitized to be processed by a micro controller. The effective sampling rate of each node is $1 \mathrm{~Hz}$. The nodes are connected to a master unit via a serial bus communication which enables synchronization of the nodes.

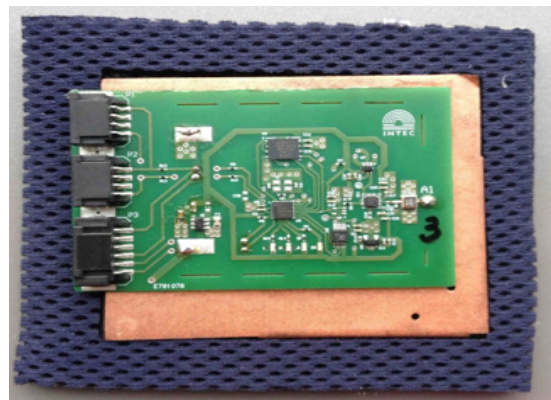

Fig. 1 Example of a receiver node integrated into a textile antenna.

\subsection{On-body setup}

The goal of this paper is to study the effect of antenna polarization on the measurement uncertainty of the BWDM and thus the BWDM is calibrated on four subjects. Eight antennas ( 2 antennas per frequency band) are placed on body on the locations that are determined to lead to the lowest measurement uncertainty using the calibration setup described in the next section (2.4). The calibration is performed on a human subject denoted as sb1 in this paper (see Table 2). The detailed procedure is presented in [15]. Table 2 lists characteristics of the subjects who participated in this study. During the calibration measurements, the optimized locations (see Figure 2) for sb1 are considered for all the subjects. Both vertical (V) and horizontal $(\mathrm{H})$ polarizations of the nodes on body are examined for the four subjects. In this paper, the terms $\mathrm{V}$ and $\mathrm{H}$ polarizations of the BWDM's nodes refer to the orientation of the antennas. As mentioned in Section 2.1 the antennas have different AR values. Therefore, the antennas are placed in two orthogonal orientations w.r.t the major/minor axis of the antennas.

Table 2 Characteristics of the subjects participating in measurements.

\begin{tabular}{lllll}
\hline Subject & $\mathrm{sb} 1$ & $\mathrm{sb} 2$ & $\mathrm{sb} 3$ & $\mathrm{sb} 4$ \\
\hline Gender & $\mathrm{M}$ & $\mathrm{M}$ & $\mathrm{M}$ & $\mathrm{M}$ \\
Age & 28 & 28 & 27 & 25 \\
Height $(\mathrm{cm})$ & 183 & 191 & 183 & 183 \\
Weight $(\mathrm{kg})$ & 76 & 84 & 70 & 73 \\
BMI $\left(\mathrm{kg} / \mathrm{m}^{2}\right)$ & 22.7 & 23.03 & 20.9 & 21.8 \\
\hline
\end{tabular}




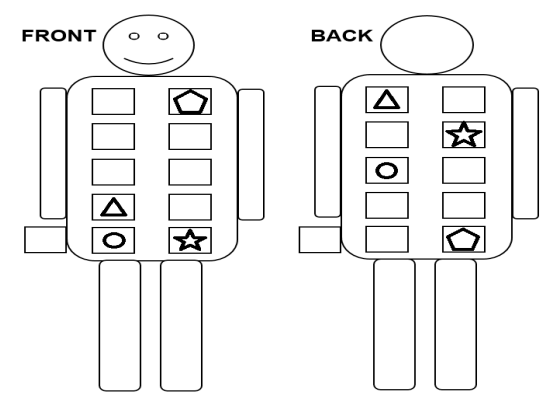

Fig. 2 The optimized locations of the nodes for each frequency band. 900-UL: circle; 900-DL: triangle; WiFi-2G: star; 2600-DL: pentagon.

\subsection{On-body calibration}

Fig. 3 shows a flow-graph of proposed on-body calibration measurements. The goal of onbody calibration is the following: First, to compare measured fields in the presence and absence of the body and to determine the measurement uncertainty of the BWDM. Second, to study the influence of antenna polarization as a function of body morphology on the measurement uncertainty of the BWDM.

First, each subject ( $\mathrm{sb} 1$ to $\mathrm{sb} 4$ ) wearing the BWDM is placed individually on a rotational platform in the far field of a standard gain horn antenna (TX) in an anechoic chamber. Each subject is rotated over $360^{\circ}$ at an angular speed of $2^{\circ}$ per second. During each rotation, the received powers $P_{r}$ are registered on each node for two (V: vertical; H: Horizontal) polarizations of the TX. This results in the received powers $P_{r}^{V}$ and $P_{r}^{H}$ on each node, where $\mathrm{H}$ and $\mathrm{V}$ are polarizations of the TX. Next, the calibration is performed for both the $\mathrm{V}$ - and $\mathrm{H}$ polarizations of the receiver nodes for each subject. Our measurement setup does not allow to measure other incident polar angles $\theta$. Therefore, the analysis of the measured data is limited to $\theta=90^{\circ}$.

Second, using an isotropic field meter (Narda NBM-550) with an EF 0391 probe the incident power density is measured in free space (in the absence of the subject) along a vertical line at the subject's locations in the range of $63-201 \mathrm{~cm}$. The aim is to measure the full body exposure; therefore, the free-space incident power density is measured up to $2 \mathrm{~m}$. It must be noted that $63 \mathrm{~cm}$ is the minimum height of the isotropic probe. In reality, the field in a given location is the result of multipath components so that its magnitude must be assessed from all field components. If the subject is facing the TX, a front antenna will measure two E field components: e.g. $E_{z}$ and $E_{x}$ for V-and H-polarizations of the TX, respectively, but if the subject is rotated over $90^{\circ}$, then the polarization the RX antenna is also rotated over $90^{\circ}$, i.e. now the antenna measures $E_{y}$ instead of Ex. For all the angles in between the antenna sees a combination of $E_{x}$ and $E_{y}$, like it would see in multipath exposure. Moreover, the broadband field meter is used to correct the measurements of the BWDM for presence of the body as well as the magnitude of the field components measured by the BWDM. The measurements are performed for both $(\mathrm{H}$ and $\mathrm{V})$ polarizations of the TX.

Third, at each frequency band, these measurements are combined to determine an effective on-body antenna aperture (AA) for each antenna as:

$$
A A^{X}(\varphi, \psi)=\frac{P_{r}^{H X}}{S_{\text {free }}^{H}} \cos ^{2}(\psi)+\frac{P_{r}^{V X}}{S_{\text {free }}^{V}} \sin ^{2}(\psi)
$$


where $X$ is the $\mathrm{V}$ or H polarization of each receiver node. $S_{\text {free }}^{H}$ and $S_{\text {free }}^{V}$ are the measured free-space power density for $\mathrm{H}$ and $\mathrm{V}$ polarizations of the TX, respectively, averaged over the different heights. $\psi$ is the polarization of an incident electric field. In a real multipath environment no assumption can be made about incident polarization. In order to determine the AA for any realistic polarization 1000 samples of $\psi$ are drawn from a uniform distribution in the range of $[0,2 \pi]$ and $A A^{X}(\varphi, \psi)$ is determined for any realistic polarization and azimuth angle, simultaneously. For each distribution the $16 \%, 50 \%, 84 \%$ percentiles are determined. This is repeated 100 times and the medians are determined for these repetitions and are denoted as $p_{16}, p_{50}$ and $p_{84}$, respectively. In a real environment the incident power density can be calculated from the received power $P_{r}^{\text {meas }}$ on the nodes as:

$$
S_{\text {inc }}=\frac{P_{r}^{\text {meas }}}{p_{50}\left(A A^{X}(\varphi, \psi)\right)}
$$

For each band, the measurement uncertainty of the BWDM is defined in terms of the median $68 \%$ confidence interval $p_{50}\left(C_{68}\right)$ of the on-body AA for the geometric average over the antennas on the front and the back. The median is determined for the above-mentioned 100 repetitions. The aim of geometric averaging over front and back is to minimize the anisotropy of the BWDM and to reduce the impact of body shadowing on the incident electric fields on the body.

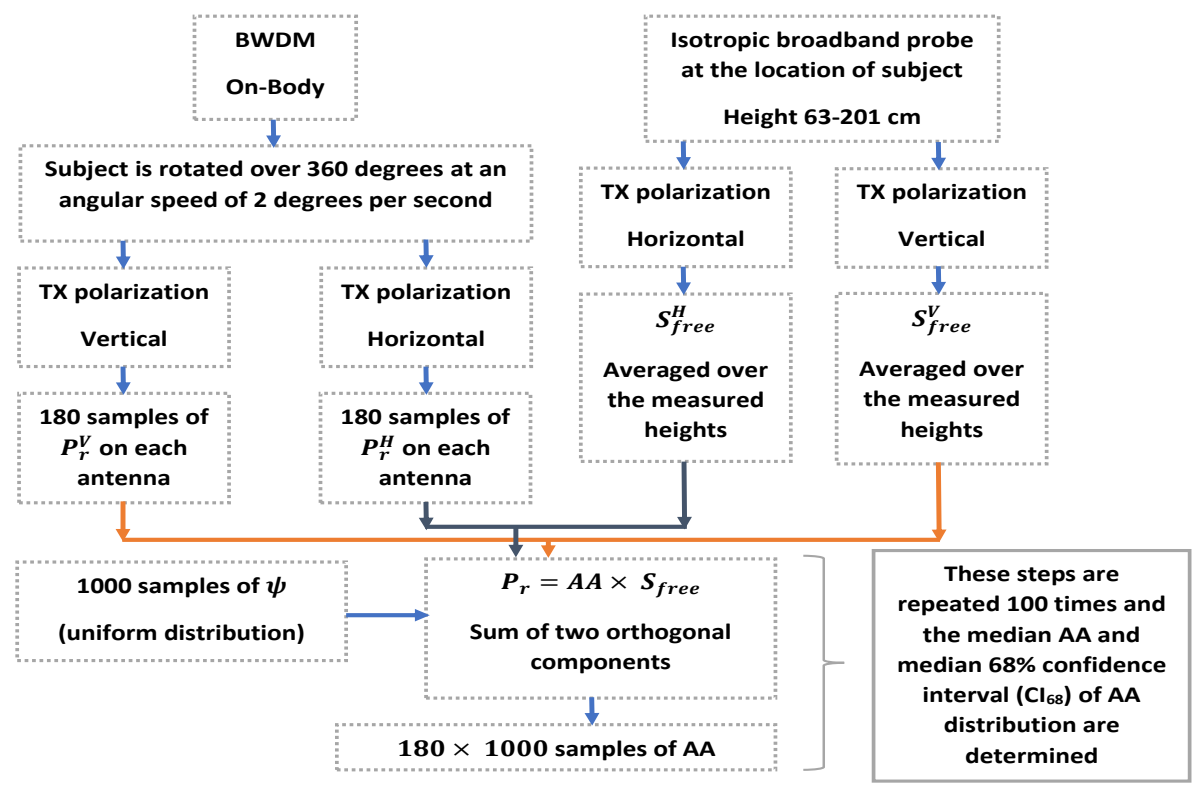

Fig. 3 Flow-graph of proposed on-body calibration measurements.

\subsection{Comparison of BDWM with ExpoM in an outdoor environment}

The applicability of the BWDM for real life exposure measurements as well as its measurement uncertainty are assessed. A commercially available PEM, ExpoM (Fields at Work 
$\mathrm{GmbH}$, Zürich, Switzerland) is used in this study to compare the measurements of the BWDM in a real environment. The ExpoM measures 16 telecommunication bands from FM radio to WiFi $5 \mathrm{GHz}$. The detection limit of the ExpoM is in the range of 0.005 to $0.05 \mathrm{~V} / \mathrm{m}$. The ExpoM is calibrated on the left hip of sb1 for the 900-DL band following a similar approach mentioned in Section 2.4 of this paper. The details of this calibration are presented in Section 2.5.2 of [15]. The goal of on-body calibration is to correct the measurements of the ExpoM for body shadowing.

Sb1 wearing the BWDM and an ExpoM on the left hip walks along a predefined route in a suburban residential area around the environment of Ghent University in Zwijnaarde science park in Belgium. The route is $1 \mathrm{~km}$ long and lasts 15 minutes. The ExpoM measures the electric fields every four seconds. Here, 900 -DL is considered since this is a dominant signal that was present outdoors during this measurement. During the walk the received powers are registered by the nodes on-body for the studied frequency bands. The on-body AA values are then used to calculate $S_{\text {inc }}$ for each frequency band. Measurements of the ExpoM have been corrected by the correction factor obtained from on-body calibration. Fig. 4 shows the predefined route for the measurement in a suburban residential area in Ghent.

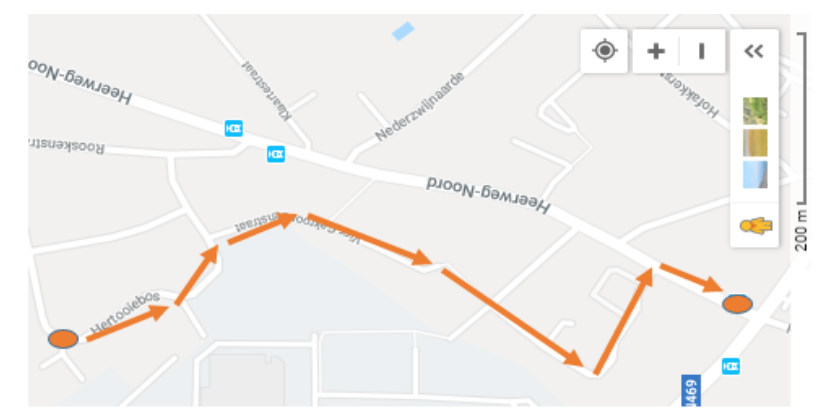

Fig. 4 The predefined route for the measurement in a suburban residential area in Ghent. Arrows show direction of the walk. (Map data @ 2018 Google.)

\section{Results}

3.1 On-body antenna aperture

Figure 5 depicts the on-body antenna apertures (AA) for the front nodes on each subject (four subjects in total) in the studied frequency bands. For 900-UL, the V antenna on the front $\left(\mathrm{F}^{V}\right)$ has a higher AA than the $\mathrm{H}$ antenna at the same location $\left(\mathrm{F}^{H}\right)$. There are also differences when comparing the different subjects.

For Sb1 AA values are $18.04 \mathrm{~cm}^{2}$ and $10.29 \mathrm{~cm}^{2}$ for configurations $\mathrm{F}^{V}$ and $\mathrm{F}^{H}$, respectively, while these values are $10.72 \mathrm{~cm}^{2}$ and $3.14 \mathrm{~cm}^{2}$, for configurations $\mathrm{F}^{V}$ and $\mathrm{F}^{H}$ on sb3, respectively. This corresponds to differences of factors 1.75 and 3.41, for sb1 and sb3, respectively. For $900-\mathrm{DL}$ and $\mathrm{WiFi}-2 \mathrm{G}, \mathrm{F}^{H}$ results in higher AA values. Sb1 has the highest difference in AA between the two antenna polarizations in the 900-UL frequency band for the antennas on the front a factor of 1.75, while the smallest difference in AA between the two antenna polarizations is observed for sb4 in the 900-DL frequency band for the same antennas on the front a factor of 1.19 . 
Increasing the frequency reduces the difference between AA values for different polarization of the antenna on the front for all studied subjects. In the 2600-DL frequency band, disregarding sb4 for which $\mathrm{F}^{V}$ has $\mathrm{AA}$ of 1.7 times larger than the $\mathrm{AA}$ of $\mathrm{F}^{H}$ on the same location, we observe small differences in the AA value.

Figure 6 shows the AA values for the two polarizations of the antenna on the back of the torso for four subjects. For $\mathrm{sb} 1$ the $\mathrm{B}^{V}$ antenna has higher AA values than $\mathrm{B}^{H}$ on the back for 900-DL and WiFi-2G. The maximum difference for sb1 occurs for 2600-DL $\left(\mathrm{B}^{V}: 4.45 \mathrm{~cm}^{2}\right.$ vs. $\mathrm{B}^{H}: 7.24 \mathrm{~cm}^{2}$ ). For $\mathrm{sb} 2, \mathrm{~B}^{V}$ has a higher $\mathrm{AA}$ than $\mathrm{B}^{H}$ which are factors of 3.7 and 1.6 times larger for 900-UL and 900-DL, respectively. Similar results are obtained in 900-DL band for all the subjects that is the $\mathrm{B}^{H}$ has a larger AA than $\mathrm{B}^{V}$.
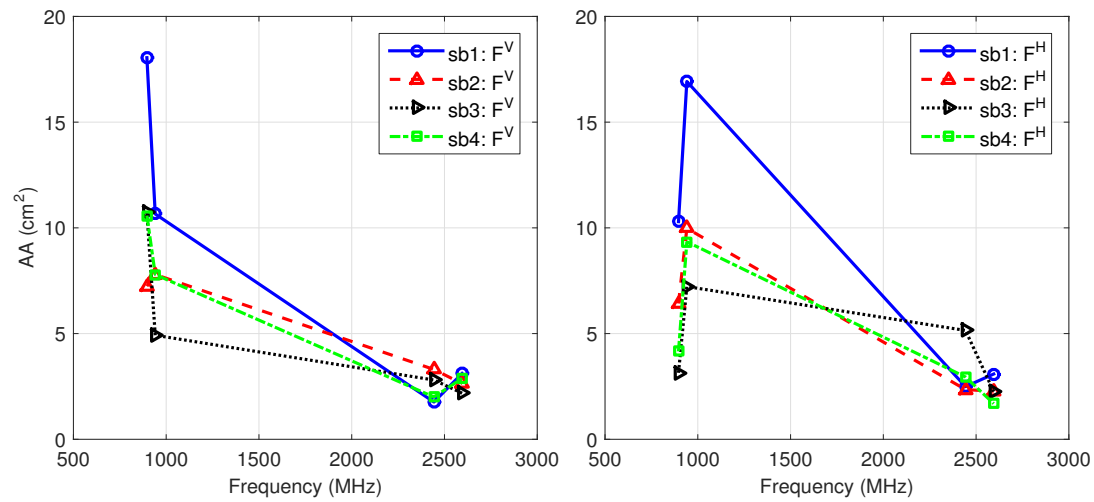

Fig. 5 The on-body antenna aperture for vertical and horizontal polarizations of the antennas on the front of the body obtained from on-body calibration of the BWDM on the four subjects. Sb1-4: subjects 1 to 4 ; F: front, B: back; V and $\mathrm{H}$ are vertical and horizontal polarization of the nodes; AA: antenna aperture.
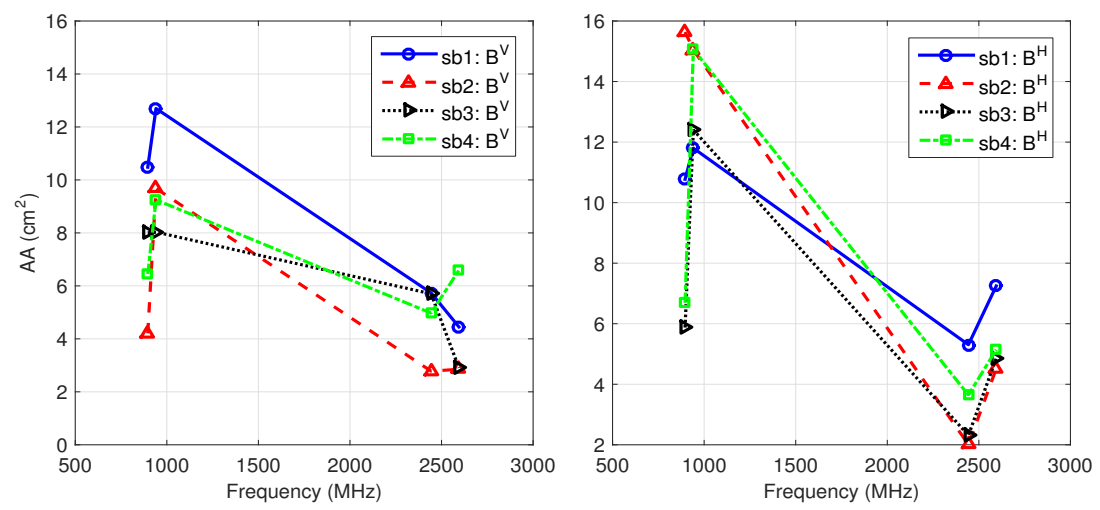

Fig. 6 The on-body antenna aperture for vertical and horizontal polarizations of the antennas on the back of the body obtained from on-body calibration of the BWDM on the four subjects. Sb1-4: subjects 1 to 4; F: front, B: back; $\mathrm{V}$ and $\mathrm{H}$ are vertical and horizontal polarization of the nodes; AA: antenna aperture. 


\section{$3.268 \%$ confidence interval of the BWDM's response}

Figure 7 illustrate the median $C I_{68}$ of the on-body antenna aperture for four subjects and different polarizations of the receiver nodes for the studied frequency bands. We disregarded configurations that have the same polarization on the front and back of the body, since these would results in lower polarization diversity. For each frequency band, these values are determined for a geometrically average over the antennas on the front and the back of the body. The standard deviations of the $C I_{68}$ (are shown with error bars in Fig. 7) is limited to $0.22 \mathrm{~dB}$ that shows the reliability of the data and also the implemented method to determine the on-body antenna aperture for each subject. For 900-UL (Fig. 7a), sb1 has the minimum $\mathrm{CI}_{68}$ of $4.94 \mathrm{~dB}$ for the $\mathrm{V}$ polarization of the node on the front and $\mathrm{H}$ polarization of the node on the back. The same configuration for other subjects results in a $C I_{68}$ of $6.1 \mathrm{~dB}(\mathrm{sb} 3)$ to $8.6 \mathrm{~dB}$ (sb2). As Fig. 7a shows, the optimal configuration for one subject (sb1) in the 900-UL band $\left(\mathrm{F}^{V}\right.$ and $\left.\mathrm{B}^{H}\right)$ is not necessarily the optimal configuration for all the other subjects. This indicates the necessity of an on-body calibration for all subjects.

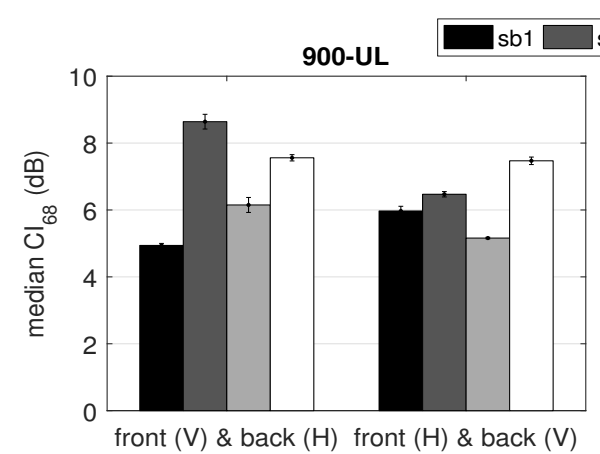

(a)

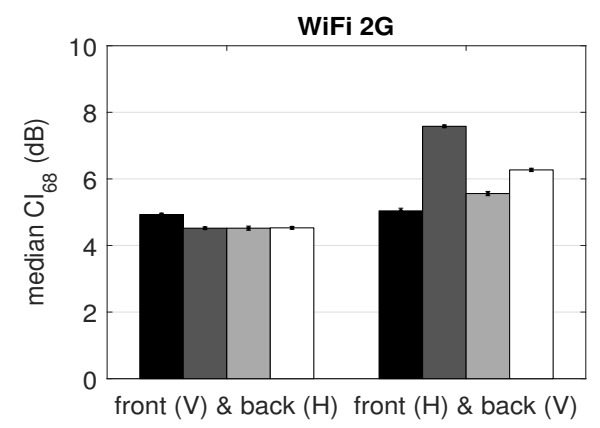

(c)

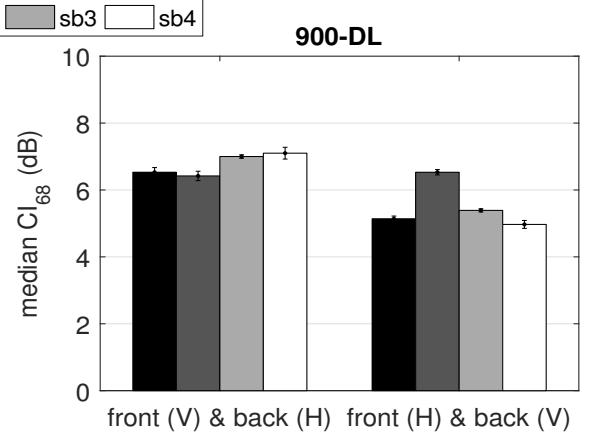

(b)

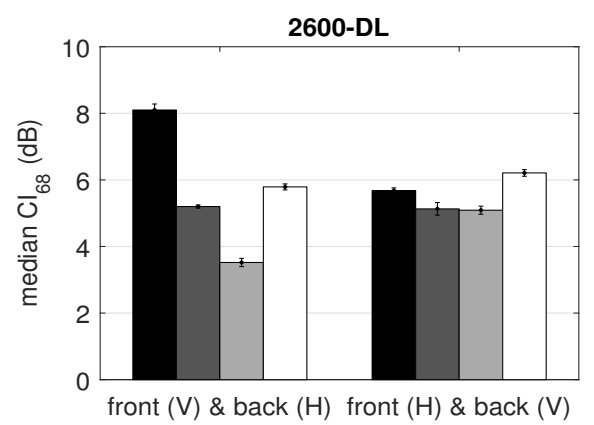

(d)

Fig. 7 Median 68\% confidence interval of the on-body antenna aperture for four studied bands, four subjects and different polarizations of the nodes on body. Error bars represent the standard deviations of the $C I_{68}$.

For 900-DL (Fig. 7b), $\mathrm{F}^{H}$ and $\mathrm{B}^{V}$ lead to the minimum $C I_{68}$ for all the subjects in the range of $4.97 \mathrm{~dB}(\mathrm{sb} 4)$ to $6.53 \mathrm{~dB}(\mathrm{sb} 2)$. Similar results are obtained for WiFi-2G (see Fig. 7c) where $\mathrm{F}^{V}$ and $\mathrm{B}^{H}$ result in the lowest $C I_{68}$ for all subjects. The range of $C I_{68}$ varies from $4.52 \mathrm{~dB}$ (sb2 and 3) to $4.93 \mathrm{~dB}$ (sb1). Considering 2600-DL (Fig. 7d), $\mathrm{F}^{H}$ and $\mathrm{B}^{V}$ results 
in the lowest $C I_{68}$ for subjects sb1 and sb2. The $C I_{68}$ in this configuration ranges from 5.09 (sb3) to 6.21 (sb4).

Table 3 lists the median $\mathrm{CI}_{68}$ for $\mathrm{VH}$ and $\mathrm{HV}$ polarizations of the nodes on the front and back of the torso for $\mathrm{sb1}$. Changing the polarizations of the nodes on the front and back for sb1 results in a difference in $C I_{68}$ in the range of $0.11 \mathrm{~dB}$ (WiFi-2G) to $2.42 \mathrm{~dB}$ (2600-DL). Using the optimized polarization for sb1 in each frequency band results in a different $C I_{68}$ in the range of $0.17 \mathrm{~dB}$ (sb4: 900-UL) to $3.7 \mathrm{~dB}$ (sb2: 900-DL). Also, for subjects with the same height $(\mathrm{sb} 1, \mathrm{sb} 3, \mathrm{sb} 4)$ the results are not consistent. However, it seems that using the optimized polarizations for sb1, when the BWDM is worn other subjects with the same height the difference in $\mathrm{CI}_{68}$ is maximum $0.59 \mathrm{~dB}$ (except 900-DL).

Table 3 Comparison of antenna polarization and $C I_{68}$ for sb1. F: Front; B: Back; $\Delta C I_{68}$ : difference between $\mathrm{F}^{V}, \mathrm{~B}^{H}$ and $\mathrm{F}^{H}, \mathrm{~B}^{V}$ for sb1. The optimized $C I_{68}$ are in bold. All values are in $\mathrm{dB}$.

\begin{tabular}{lllllll}
\hline RF signal & $\mathrm{F}_{s b 1}^{V}, \mathrm{~B}_{s b 1}^{H}$ & $\mathrm{~F}_{s b 1}^{H}, \mathrm{~B}_{s b 1}^{V}$ & $\Delta C I_{68}$ & $\Delta \mathrm{sb2}$ & $\Delta \mathrm{sb3}$ & $\Delta \mathrm{sb4}$ \\
\hline 900-DL & 6.53 & $\mathbf{5 . 1 4}$ & 1.39 & 3.7 & 1.21 & 2.62 \\
900-UL & $\mathbf{4 . 9 4}$ & 5.97 & 1.03 & 1.39 & 0.25 & -0.17 \\
WiFi-2G & $\mathbf{4 . 9 3}$ & 5.04 & 0.11 & -0.41 & -0.41 & -0.4 \\
2600-DL & 8.1 & $\mathbf{5 . 6 8}$ & 2.42 & -0.55 & -0.59 & 0.53 \\
\hline
\end{tabular}

\subsection{Comparison of BDWM with ExpoM in an outdoor environment}

Fig. 8 depicts the measured $S_{\text {inc }}$ values during the 15-minute walk by the BWDM and the ExpoM. The results show that behaviors of the measured $S_{i n c}$ values by both devices are similar (e.g. peaks). The summary statistics are listed in Table 4 . The measured mean $S_{\text {inc }}$ are 70.1 and $83.8 \mu \mathrm{W} / \mathrm{m}^{2}$ for the BWDM and the ExpoM, respectively. Median $S_{\text {inc }}$ of 39 and $41 \mu W / m^{2}$ are registered by the BWDM and the ExpoM, respectively. This is a very good agreement between $p 50$ of $S_{i n c}$ for the BWDM compared to the ExpoM. The measured data is in the range of $3.51 \mu \mathrm{W} / \mathrm{m}^{2}$ to $1.5 \mathrm{~mW} / \mathrm{m}^{2}$ for the BWDM while the ExPoM registered a range of 2.85 to $1 \mathrm{~mW} / \mathrm{m}^{2}$. Both devices registered no data below their detection limits. All the measurements are below the issued reference levels by ICNIRP [19]. Considering an AA of $12.71 \mathrm{~cm}^{2}$ for sb1 (for the average over front and back) in the 900-DL band, the BWDM has a detection limit of $0.13 \mu \mathrm{W} / \mathrm{m}^{2}$ while ExpoM has a detection limit of $0.07 \mu \mathrm{W} / \mathrm{m}^{2}$ in the same frequency band. The optimized nodes for sb1 result in a $C_{68}$ of $5.14 \mathrm{~dB}$ which is $7.5 \mathrm{~dB}$ lower than $C I_{68}$ of the ExpoM calibrated on the same subject [15].

\section{Discussion}

For the single antennas on body, the obtained antenna apertures do not show an evident relationship with the BMI. Moreover, similar frequency dependencies for the different subjects for the same configurations on the body are found. For incident RF EMFs on body, part of the waves are either absorbed or scattered by the body and the remaining part propagates toward the nodes. The findings of [4] indicate a higher attenuation due to the body for vertical polarization at $2.4 \mathrm{GHz}$. In this paper, for $\mathrm{WiFi}-2 \mathrm{G}(2443 \mathrm{MHz})$ the horizontal antenna on the front resulted in higher AA values for all the subjects. These results are consistent for the $\mathrm{H}$ 


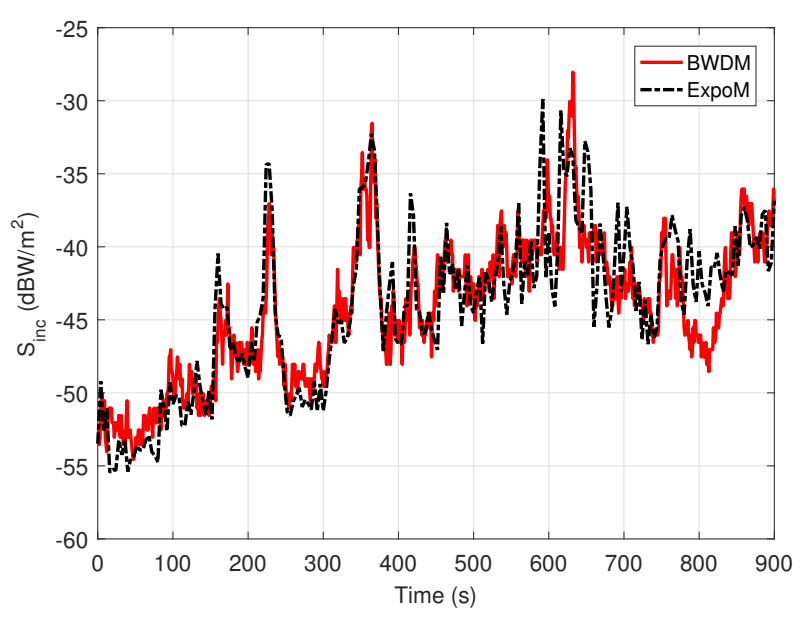

Fig. 8 The incident power density measured during the walk for 900-DL. Both measurements of BWDM and ExpoM has been corrected using calibration factors.

Table 4 Summary statistics of the measured 900-DL during a 15-minute walk for the BWDM and the ExpoM. ${ }^{*}$ The polarizations of the nodes are optimized for sb1 and has the minimum $C I_{68}$. All the values are in $\left(\mu W / m^{2}\right)$ unless indicated.

\begin{tabular}{lll}
\hline$S_{\text {inc }}$ & BWDM $\left(\mu W / m^{2}\right)$ & $\operatorname{ExpoM}\left(\mu W / m^{2}\right)$ \\
\hline$\mu$ & 70.1 & 83.8 \\
$\sigma$ & 114.7 & 128.93 \\
p25 & 15.68 & 16.33 \\
p50 & 39.41 & 41.28 \\
p75 & 88.22 & 90.77 \\
min & 3.51 & 2.85 \\
max & 1500 & 1000 \\
detection limit & 0.13 & 0.07 \\
\hline nodes* & Front ${ }^{*}$, Back $^{V}$ & - \\
AA $\left(\mathrm{cm}^{2}\right)$ & 12.71 & - \\
$C I_{68}(\mathrm{AA})(\mathrm{dB})$ & 5.14 & $12.64[15]$ \\
\hline
\end{tabular}

antenna on the back of all the subjects in the 900-DL band. In addition, the existing literature are not conclusive regarding the polarization dependency and the absorption of the EMFs and thus a general agreement cannot be found. A higher absorption for horizontally polarized incident fields has been previously reported at frequencies higher than $2 \mathrm{GHz}$ [20,21]. Similarly, some studies obtained a higher absorption for vertical polarization of the incident plane waves [22].

The results show that optimizing the polarization of the nodes on one subject could result in higher confidence intervals of the on-body antenna aperture for the subjects with different body morphologies below $0.5 \mathrm{~dB}$ and up to $2.62 \mathrm{~dB}$ for subjects with the same height and subjects with different height, respectively. For example for 900-UL, when the BWDM with the optimized configuration for sb1 (front ${ }^{V}$, back ${ }^{H}$ ) is worn by sb2, the $C I_{68}$ increases from 4.94 to $8.64 \mathrm{~dB}$ (difference of $3.7 \mathrm{~dB}$ ). The height, weight and BMI of sb2 are $4.37 \%, 10.52 \%$ and $1.45 \%$ higher than sb1. Similarly, in 2600-DL band, sb1 wearing the BWDM optimized for sb3 (front ${ }^{V}$, back ${ }^{H}$ ), has an increased $C I_{68}$ of $4.94 \mathrm{~dB}$. This is while both the subjects 
have a similar height, but the weight and BMI of sb1 are $4.1 \%$ larger than sb4. It is not clear whether a specific antenna polarization on the front and the back (e.g. V on the front and $\mathrm{H}$ on the back) could result in a higher measurement uncertainty.

From the results we conclude that the location of the nodes on body have a higher influence on the directive gain of the antennas and consequently the measurement uncertainty of the BWDM (up to $3.7 \mathrm{~dB}$ ) rather than the height or BMI of the subjects. The e results are based on the limited range of BMI/height in this study. Study of male and female subjects with higher variations in height, BMI and age would be part of the future research. The BWDM has been calibrated on sb1 for WiFi-2G and 2600-DL bands in a reverberation chamber (RC) [23]. The locations of the nodes are optimized based on the calibration in the RC. The results showed that the optimized location of the nodes for the BWDM based on calibrations in the anechoic chamber are in excellent agreement with those obtained from calibration measurements in RC. Therefore, the optimized locations obtained from measurements in the anechoic chamber are not purely random for each subject.

The authors suggest to use antennas with dual polarization which register the orthogonal components of the RF fields with one antenna. In this case the orientation of the antenna with respect to the human body is less critical. For determination of the antenna aperture used for each pair of antennas, a random polarization has been taken into account. The $C I_{68}$ values already contain a dependency on polarization during rotation in the azimuth. So the antenna apertures underestimate waves with polarization mismatch and overestimate waves with little or no polarization mismatch. It must be noted that for the BWDM during optimization of the polarization setup, all options ( $\mathrm{VV}, \mathrm{HV}, \mathrm{VH}, \mathrm{HH}$ on the front and back) were tested and best combination (with minimum $\mathrm{CI}_{68}$ ) is chosen (the one with the least dependence on polarization and azimuth).

Since all measurements were performed using the same equipment and in similar environmental conditions, the uncertainty differences due to the fabrication tolerances, deformations during operation, humidity and temperature, receiver(s) electronics (non-linearity, frequency selectivity, temperature drift) are small compared to the uncertainty due to body shadowing and different body morphologies. In addition, the goal of this paper is to study the effect of body morphology on the measurement uncertainty of the BWDM. Using EF 0391 probe in combination with the NBM-550 field meter causes a combined standard uncertainty of $1.1 \mathrm{~dB}$ on the measurements including linearity, isotropic and thermal responses and frequency sensitivity [24].

The outdoor measurement of both BWDM and ExpoM are in good agreement. Considering the detection limits of both devices the detection limit of the ExpoM is a factor of 1.85 lower than the on-body detection limit of the BWDM worn by sb1. However, single ExpoM has a larger measurement uncertainty $(7.5 \mathrm{~dB}$ larger) in this paper. Factors larger than 2 have been reported in the literature $[4,6]$ for the underestimation of the PEMs.

\section{Conclusions}

For the first time, the polarization dependency of a multi-band body-worn distributed exposure meter (BWDM) and the effect of body morphology on this dependency is assessed. The BDWM consists of 8 nodes measuring at four frequency band including downlink (DL) and uplink bands of $900 \mathrm{MHz}, \mathrm{WiFi} 2 \mathrm{GHz}$ and DL band of $2600 \mathrm{MHz}$. The BDWM is calibrated on four male subjects with different body mass indexes (BMIs). For each subject the on-body antenna aperture and the uncertainty of the BWDM is determined for vertical and horizontal polarizations of the nodes in each of the above frequency bands. The obtained 
antenna apertures do not show an evident relationship with the BMI. Moreover, the results show that optimizing the polarization of the nodes on one subject could result in higher confidence interval values of the on-body antenna aperture for the subjects with different body morphologies. This is less than $0.5 \mathrm{~dB}$ and up to $2.62 \mathrm{~dB}$ for subjects with the same height and subjects with different height, respectively. From the results we conclude that the location of the nodes on body have a higher influence up to $3.7 \mathrm{~dB}$ on the directive gain of the antennas and consequently the measurement uncertainty of the BWDM rather than the height or BMI of the subjects. This could increase up to $4.9 \mathrm{~dB}$ if the locations of the nodes are not optimized on body. The authors suggest to use antennas with dual polarization which register the orthogonal components of the RF fields with one antenna. As an application, a median power density of $39 \mu \mathrm{W} / \mathrm{m}^{2}$ is registered by the BWDM in a suburban residential area in Ghent which is below the issued reference levels by ICNIRP.

Acknowledgements This research was funded by the Research Foundation Flanders (FWO) under grant agreement No G003415N and the French National Research Program for Environmental and Occupational Health of ANSES (2015/2 RF/07) as part of project ACCEDERA. A.T. has received funding from the European Union's Horizon 2020 research and innovation programme under the Marie Skłodowska-Curie grant agreement No 665501 with the FWO. A.T. is an FWO [PEGASUS] ${ }^{2}$ Marie Skłodowska-Curie Fellow. S.A. is a Post-Doctoral Fellow of the FWO.

\section{References}

1. World Health Organization (WHO). Radio frequency (RF) fields research agenda. http://www. who int/peh-emf/research/agenda/en/, accessed on 15 February 2018.

2. M. Eeftens, B. Struchen, L.E. Birks, E. Cardis, M. Estarlich, M.F. Fernandez, P. Gajšek, M. Gallastegi, A. Huss, L. Kheifets, I.K. Meder, J. Olsen, M. Torrent, T. Trček, B. Valič, R. Vermeulen, M. Vrijheid, L. van Wel, M. Guxens, M. Röösli, Personal exposure to radio-frequency electromagnetic fields in europe: Is there a generation gap?, Environment International 121, 216 (2018). DOI https://doi.org/10.1016/j. envint.2018.09.002

3. K. Gryz, P. Zradziński, J. Karpowicz, The role of the location of personal exposimeters on the human body in their use for assessing exposure to the electromagnetic field in the radiofrequency range 98-2450 mhz and compliance analysis: evaluation by virtual measurements, BioMed research international 2015 (2015)

4. J.F. Bolte, G. van der Zande, J. Kamer, Calibration and uncertainties in personal exposure measurements of radiofrequency electromagnetic fields, Bioelectromagnetics 32(8), 652 (2011)

5. G. Thuróczy, F. Molnár, G. Jánossy, N. Nagy, G. Kubinyi, J. Bakos, J. Szabó, Personal rf exposimetry in urban area, Annals of Telecommunications-annales des télécommunications 63(1-2), 87 (2008)

6. A. Thielens, S. Agneessens, L. Verloock, E. Tanghe, H. Rogier, L. Martens, W. Joseph, On-body calibration and processing for a combination of two radio-frequency personal exposimeters, Radiation Protection Dosimetry 163(1), 58 (2015)

7. J.F. Bolte, Lessons learnt on biases and uncertainties in personal exposure measurement surveys of radiofrequency electromagnetic fields with exposimeters, Environment International 94(Supplement C), 724 (2016). DOI https://doi.org/10.1016/j.envint.2016.06.023

8. A. Bahillo, J. Blas, P. Fernández, R.M. Lorenzo, S. Mazuelas, E.J. Abril, E-field assessment errors associated with rf dosemeters for different angles of arrival, Radiation Protection Dosimetry 132(1), 51 (2008)

9. G. Neubauer, S. Cecil, W. Giczi, B. Petric, P. Preiner, J. Fröhlich, M. Röösli, The association between exposure determined by radiofrequency personal exposimeters and human exposure: A simulation study, Bioelectromagnetics 31(7), 535 (2010)

10. S. de Miguel-Bilbao, V. Ramos, J. Blas, Assessment of polarization dependence of body shadow effect on dosimetry measurements in $2.4 \mathrm{ghz}$ band, Bioelectromagnetics 38(4), 315 (2017). DOI 10.1002/bem. 22030

11. A.N. López, J. Gonzalez-Rubio, J.M.V. Montoya, E.A. Garde, Using multiple exposimeters to evaluate the influence of the body when measuring personal exposition to radio frequency electromagnetic fields, COMPEL - The international journal for computation and mathematics in electrical and electronic 
engineering 34(4), 1063 (2015). DOI 10.1108/COMPEL-10-2014-0268. URL https://doi .org/10. 1108/COMPEL-10-2014-0268

12. R. Aminzadeh, A. Thielens, A. Bamba, L. Kone, D.P. Gaillot, M. Lienard, L. Martens, W. Joseph, Onbody calibration and measurements using personal radiofrequency exposimeters in indoor diffuse and specular environments, Bioelectromagnetics 37(5), 298 (2016)

13. P. Vanveerdeghem, P. Van Torre, A. Thielens, J. Knockaert, W. Joseph, H. Rogier, Compact personal distributed wearable exposimeter, IEEE Sensors Journal 15(8), 4393 (2015)

14. A. Thielens, S. Agneessens, H. De Clercq, J. Lecoutere, L. Verloock, E. Tanghe, S. Aerts, R. Puers, H. Rogier, L. Martens, W. Joseph, On-body calibration and measurements using a personal, distributed exposimeter for wireless fidelity, Health Phys 108(4), 407 (2015)

15. R. Aminzadeh, A. Thielens, S. Agneessens, P. Van Torre, M. Van den Bossche, S. Dongus, M. Eeftens, A. Huss, R. Vermeulen, R. de Seze, P. Mazet, E. Cardis, H. Rogier, M. Röösli, L. Martens, W. Joseph, A multi-band body-worn distributed radio-frequency exposure meter: Design, on-body calibration and study of body morphology, Sensors 18(272) (2018). DOI 10.3390/s18010272

16. A. Thielens, P. Vanveerdeghem, P. Van Torre, S. Gängler, M. Röösli, H. Rogier, L. Martens, W. Joseph, A personal, distributed exposimeter: Procedure for design, calibration, validation, and application, Sensors 16(2) (2016)

17. A. Thielens, L. Martens, W. Joseph, Comments on assessment of polarization dependence of body shadow effect on dosimetry measurements in $2.4 \mathrm{GHz}$ band, Bioelectromagnetics 38(8), 648 (2017). DOI 10.1002/bem. 22080

18. S. Agneessens, Coupled eighth-mode substrate integrated waveguide antenna: Small and wideband with high-body antenna isolation, IEEE Access (2017)

19. International Commission on Non-ionizing Radiation Protection (ICNIRP), Guidelines for limiting exposure to time-varying electric, magnetic, and electromagnetic fields (up to $300 \mathrm{GHz}$ ), Health Phys. 74(4), 494 (1998)

20. A. Hirata, N. Ito, O. Fujiwara, Influence of electromagnetic polarization on the whole-body averaged sar in children for plane-wave exposures, Physics in Medicine \& Biology 54(4), N59 (2009). URL http://stacks.iop.org/0031-9155/54/i=4/a=NO2

21. A. Bamba, W. Joseph, G. Vermeeren, A. Thielens, E. Tanghe, L. Martens, A formula for human average whole-body sar wb under diffuse fields exposure in the ghz region, Physics in Medicine \& Biology 59(23), 7435 (2014). URL http: //stacks.iop.org/0031-9155/59/i=23/a=7435

22. S. Kühn, W. Jennings, A. Christ, N. Kuster, Assessment of induced radio-frequency electromagnetic fields in various anatomical human body models, Physics in Medicine \& Biology 54(4), 875 (2009). URL http: //stacks . iop. org/0031-9155/54/i=4/a=004

23. R. Aminzadeh, A. Thielens, D.P. Gaillot, M. Lienard, S. Agneessens, P.V. Torre, M.V. den Bossche, H. Rogier, M. Röösli, L. Martens, W. Joseph, in 2018 2nd URSI Atlantic Radio Science Meeting (ATRASC) (2018), pp. 1-4. DOI 10.23919/URSI-AT-RASC.2018.8471583

24. Narda Safety Test Solutions. Electric and Magnetic Field Measurement, NBM Series Probes., EF0391: Economical field probe for universal frequency range. $100 \mathrm{kHz}-3 \mathrm{GHz}$ (2016). NBM-Probes: DataSheet 